\title{
An Analysis of VLF Mode Propagation for a Variable Ionosphere Height
}

\author{
James R. Wait \\ Contribution from Central Radio Propagation Laboratory \\ National Bureau of Standards, Boulder, Colo.
}

(Received February 1, 1962)

\begin{abstract}
An approximate treatment of modes in a waveguide of variable width is presented. It is assumed that the boundaries satisfy impedance-type boundary conditions. The model consists of two parallel plate waveguide regions connected by a linearly tapered section. The results have application to the theory of VLF radio wave propagation when the ionospheric heights are not constant along the path.
\end{abstract}

\section{Introduction}

The waveguide mode theory has been quite successful in explaining many of the broad features of propagation of VLF radio waves to great distances. Furthermore, the diurnal variation of phase of a $\mathrm{cw}$ carrier can often be interpreted in terms of the relative amount of solar illumination on the great circle path. In carrying out such an interpretation, it is usually assumed that a waveguide mode passes smoothly through the day/night transition without change of field pattern across the guide. Indeed, the experimental data show little evidence of distortion of the field in this transition region. Exceptions are indicated in the data of Lauter and Sprenger [1952] who observed amplitude variations in East Germany from the GBR transmission from England which indicated an undulating field. He explained this qualitatively in terms of diffraction from a ledge of ionization which was truncated at sunrise and sunset.

An alternative approach is followed here which would appear to be more appropriate for propagation to great distances. The model chosen is a parallel plate waveguide of constant width $h$ which is connected to a waveguide of constant width $h+\Delta h$ by a linearly tapered section. The nature of the waveguide modes in the two parallel plate waveguides is well known. The character of the modes, in the tapered section, is first obtained by examining the permissible solutions in idealized wedge regions. Then an approximate method is applied to evaluate the coupling between the three waveguide regions.

\section{Wedge Solutions}

We consider a wedge consisting of two nonparallel plane surfaces. With respect to a cylindrical coordinate system $(\rho, \phi, z)$, the apex of the wedge is the $z$ axis and its surfaces are at $\phi=0$ and $\phi=\psi$. The situation is shown in figure 1. For simplicity the fields are taken

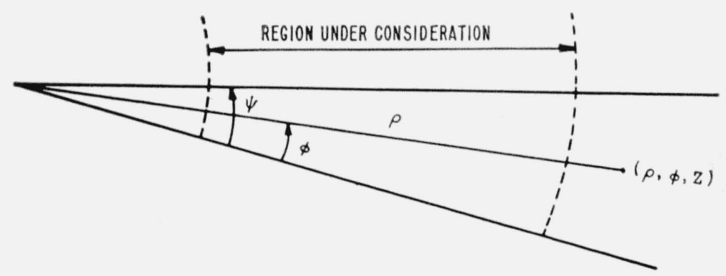

FIGURE 1. The wedge region. 
not to vary in the $z$ direction so that $\partial / \partial z=0$. This restricts the direction of propagation to be normal to the apex of the wedge region. Furthermore, the magnetic field is taken to have only a $z$ component. Since the lower surface $(\phi=0)$ is to represent the ground, it will be assumed that $E_{\rho}=0$ for $\phi=0$ and $0<\rho<\infty$. The upper boundary which is to represent the lower edge of the ionosphere is assumed to be characterized by a surface impedance $Z$. Thus

$$
E_{\rho}=-Z H_{z} \text { for } \phi=\psi \text { and } 0<\rho<\infty .
$$

A suitable solution within the region $0<\phi<\psi$ is of the form [Wait, 1959]

$$
H_{z}=A Z_{\nu}(k \rho) \cos \nu \phi
$$

where $A$ is a constant and $Z_{\nu}$ is a cylindrical Bessel function of (complex) order $\nu$ and argument $k \rho$. The choice of the factor $\cos \nu \phi$ automatically assures that the boundary condition at $\phi=0$ is satisfied.

The boundary condition at $\phi=\psi$ can be rewritten in the form ${ }^{1}$

$$
\frac{\partial H_{z}}{\partial \phi}=-i \epsilon \omega \rho Z(\rho) H_{z}
$$

where it is indicated that the surface impedance is a function of $\rho$. To facilitate the solution, it is necessary to choose the $\rho$ dependence such that

$$
Z(\rho)=Z_{0} \frac{\rho_{0}}{\rho}
$$

where $\rho_{0}$ and $Z_{0}$ are constants. Thus

$$
\frac{\partial H_{z}}{\partial \phi}=-i \epsilon \omega \rho_{0} Z_{0} H_{z} \text { at } \phi=\psi
$$

where $\epsilon$ is the dielectric constant in the wedge region. On applying eq (5) to eq (2) we see that

$$
\nu \tan \nu \psi=i \epsilon \omega \rho_{0} Z_{0}
$$

Solution of this equation yields a set of values of $\nu$ which are denoted $\nu_{s}$.

We shall now demonstrate that these modes are orthogonal over the angular domain of the wedge. For example, consider

$$
I_{s, q}=\int_{0}^{\psi} \cos \left(\nu_{s} \phi\right) \cos \left(\nu_{q} \phi\right) d \phi
$$

where $\nu_{s}$ and $\nu_{q}$ both satisfy eq (6). Since

$$
I_{s, q}=\frac{\sin \left(\nu_{s}-\nu_{q}\right) \psi}{2\left(\nu_{s}-\nu_{q}\right)}+\frac{\sin \left(\nu_{s}+\nu_{q}\right) \psi}{2\left(\nu_{s}+\nu_{q}\right)}
$$

it is not difficult to show that

$$
I_{s, q}=0 \text { if } \nu_{s} \neq \nu_{q}
$$

which demonstrates the orthogonality. The normalization constant for the modes is

$$
I_{s, s}=\int_{0}^{\psi} \cos ^{2}\left(\nu_{s} \phi\right) d \phi=\frac{\psi}{2}\left[1+\frac{\sin \left(2 \nu_{s} \psi\right)}{2 \nu_{s} \psi}\right]
$$

It is now a simple matter to express the field $H_{z}(\rho, \phi)$ at any point in the wedge in terms of its value over the surface $\rho=\rho_{1}, 0<\phi<\psi$. Since the modes form a complete set,

1 The time factor is $\exp (i \omega t)$. 


$$
H_{z}(\rho, \phi)=\sum_{s} A_{s} Z_{\nu_{s}}(k \rho) \cos \nu_{s} \phi
$$

where the summation is over all modes. Then, because of orthogonality, the coefficient $A_{s}$ for the $s$ th mode may be expressed as

$$
A_{s}=\frac{P_{s}}{Z_{\nu_{s}}\left(k \rho_{1}\right) \frac{\psi}{2}\left[1+\frac{\sin \left(2 \nu_{s} \psi\right)}{2 \nu_{s} \psi}\right]}
$$

where

$$
P_{s}=\int_{0}^{\psi} H_{z}\left(\rho_{1}, \phi\right) \cos \nu_{s} \phi d \phi
$$

Thus, if we can find a suitable approximation for $H_{z}\left(\rho_{1}, \phi\right)$ the corresponding function $H_{z}(\rho, \phi)$ can be obtained by performing an integration.

Before proceeding further it is desirable to discuss something about the properties of the modes in the wedge region. Until the source itself is prescribed, the radial or $\rho$ dependence of the field is not known. However, in general, the cylindrical Bessel function $Z_{\nu_{s}}(k \rho)$ may be represented as the linear combination of two Hankel functions, of the first and second kind, of order $\nu_{s}$. Thus a mode is of the form

$$
\left\{\begin{array}{l}
H_{v_{s}}^{(1)}(k \rho) \\
H_{v_{s}}^{(2)}(k \rho)
\end{array}\right\} \cos \nu_{s} \phi
$$

If $k \rho>>1$ and $k \rho>\nu_{s} \mid$ it is permissible to replace the Hankel functions by their Debye or second-order approximations [Wait, 1959]. Thus

$$
H_{\nu_{s}}^{(1)}(k \rho) \cong\left(\frac{2}{\pi k \rho \sin \alpha}\right)^{\frac{1}{2}} \exp [ \pm i k \rho(\sin \alpha-\alpha \cos \alpha)] e^{\mp i \pi / 4}
$$

where $\alpha$ is defined by

$$
\nu_{s}=k \rho \cos \alpha .
$$

An alternative form is

$$
H_{\nu_{s}}^{(1)}(k \rho) \cong\left(\frac{2}{\pi k \rho \sin \alpha}\right)^{\frac{1}{2}} \exp [ \pm i k \rho \sin \alpha] \exp \left[\mp i\left(\alpha \nu_{s}+\frac{\pi}{4}\right)\right] .
$$

It is evident that these have the character of traveling waves propagating in negative and positive $\rho$ directions, respectively. In this case the mode equation $\nu_{s}$ tan $\nu_{s} \psi=i \epsilon \omega \rho_{0} Z_{0}$ can be written in the form

$$
C \tan k C h^{\prime}=i Z / \eta_{0}
$$

or

$$
\frac{C-Z / \eta_{0}}{C+Z / \eta_{0}} e^{-i 2 k C h^{\prime}}=1
$$

where

$$
\begin{aligned}
& C=\cos \alpha=\nu_{s} / k \rho, \\
& Z=\frac{\rho_{0}}{\rho} Z_{0},
\end{aligned}
$$

and

$$
h^{\prime}=\rho \psi
$$

It is seen that eq (15) has precisely the form of the modal equation for a parallel place waveguide of equivalent height $h^{\prime}$ and surface impedance (on one wall) equal to $Z$ [Wait, 1960]. 
The choice of the kind of Hankel function (i.e., first or second) is dictated by the nature of the problem. In general, a linear combination is required. However, if the waves are excited over the aperture plane $\rho=\rho_{1}$ then for $\rho>\rho_{1}$ in an infinite wedge region the Hankel function of the second kind only is needed.

\section{Composite Problem}

We shall now consider the composite waveguide problem mentioned in the introduction. The situation is illustrated in figure 2 .

A Cartesian coordinate system, $(x, y, z)$, is chosen such that the earth's surface (assumed flat) is the plane $z=0$. An ionospheric reflecting layer of surface impedance $Z_{1}$ is located at $z=h$ and $x<0$. A similar reflecting layer of surface impedance $Z_{2}$ is located at $z=h+\Delta h$ for $x>b$. The transition section extends from $x=0$ to $x=b$ with a linear variation of height from $h$ to $h+\Delta h$. The surface impedance of the surface of the transition section must be a linear function of $\rho$ in order to make use of the orthogonal set of modes discussed above. Jt is convenient to choose $Z=Z_{0} \rho_{0} / \rho$ where $\rho_{0}=a$ sec $\psi$ which is equivalent to

$$
Z=Z_{0} \frac{a}{x+a} \text { for the interval } 0<x<b .
$$

A single waveguide mode is now considered to be incident from the left. It is taken to be of the form [Wait, 1960]

$$
H_{z}=e^{-i k x S_{p}} \cos k C_{p} z
$$

where $S_{p}=\left(1-C_{p}^{2}\right)^{\frac{1}{2}}$ and $C_{p}$ is a solution of

where $p=0,1,2, \ldots$ and

$$
\frac{C-\Delta_{1}}{C+\Delta_{1}} e^{-i 2 k h C}=e^{-i 2 \pi p}=1
$$

$$
\Delta_{1}=\frac{Z_{1}}{\eta_{0}}
$$

The integer $p$ is the order of the waveguide mode under consideration. A general incident field is obtained by superimposing all possible $p$ modes.

In a rigorous treatment of this problem we would need to set up a general representation of the fields in the three waveguide regions. To evaluate the unknown coefficients it is then required to match the tangential electric and magnetic fields across the aperture planes at $x=0$ and $x=b$. This leads to a very cumbersome (infinite) set of equations which must be solved for the infinite sets of coefficients. The problem is also complicated since the plane $x=$ constant is not a coordinate surface in the natural cylindrical coordinates for the wedge region.

In order to obtain a useful result, albeit approximate, a number of simplifying assumptions are made. The justification for these are mainly on physical grounds. Essentially, the idea is to neglect the reflected waves at the aperture planes at $x=0$ and $x=b$. This approach has been used by Solymar [1959] who treated a number of analogous problems in microwave guides, and by Furutsu [1957] who considered related problems in radio propagation over mixed paths.

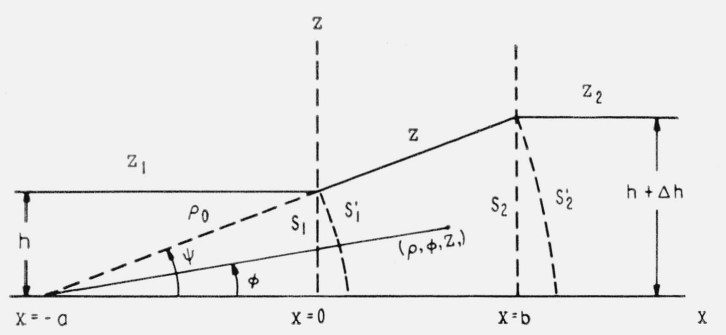

Figure 2. The transition problem (not drawn to scale since $\psi \ll 1)$. 
In view of the above assumption, the field in the wedge transition region has the form

$$
H_{z}=\sum_{s} A_{s} H_{\nu_{s}}^{(2)}(k \rho) \cos \nu_{s} \phi
$$

where $\nu_{s}$ is a solution of

$$
\nu_{s} \tan \nu_{s} \psi=i \epsilon \omega \rho_{0} Z_{0}
$$

The coefficients $A_{s}$ are given by

$$
A_{s}=\frac{P_{s}}{H_{\nu_{s}}^{(2)}\left(k \rho_{0}\right) \frac{\psi}{2}\left[1+\frac{\sin \left(2 \nu_{s} \psi\right)}{2 \nu_{s} \psi}\right]}
$$

where

$$
P_{s}=\int_{0}^{\psi} H_{z}\left(\rho_{0}, \phi\right) \cos \nu_{s} \phi d \nu
$$

If the length of the transition section, $b$, is large compared with the height change, $\Delta h$, it is reasonable to assume that $H_{z}\left(\rho_{0}, \phi\right)$ is given adequately by the incident mode. This has the form

and thus

$$
H_{z}^{\mathrm{inc}}=e^{-i k x S_{p}} \cos k C_{p} z
$$

$$
H_{z}\left(\rho_{0}, \phi\right) \cong e^{-i k x_{0} S_{p}} \cos \left(k \rho_{0} C_{p} \phi\right)
$$

where

$$
\begin{aligned}
x_{0} & =\left(\rho_{0}^{2}-z^{2}\right)^{\frac{1}{2}}-\rho_{0} \cos \psi \\
& \simeq \rho_{0}\left(\frac{\psi^{2}}{2}-\frac{\phi^{2}}{2}\right) .
\end{aligned}
$$

Therefore

$$
P_{s}^{(p)}=\int_{0}^{\psi} \cos \left(k \rho_{0} C_{p} \phi\right) \cos \left(\nu_{s} \phi\right) \exp \left[-i k S_{p} \rho_{0}\left(\frac{\psi^{2}}{2}-\frac{\phi^{2}}{2}\right)\right] d \phi
$$

where the superscript $p$ is to indicate that this is the value appropriate to the $p$ th incident mode. The function $P_{s}^{(p)}$ in the form given above may be rewritten in the form

$$
P_{s}^{(p)}=e^{-i k S_{p} \rho_{0} \psi^{2} / 2} I\left[k \rho_{0} C_{p}, \nu_{s}, k \rho_{0} S_{p} / 2, \psi\right]
$$

where the integral $I$ of the four variables, is expressible in terms of Fresnel integrals (see appendix). This is restricted only by the previously imposed condition that $\psi<<1$.

A somewhat simpler approach is possible if, in addition, $k \rho_{0} \psi^{2}<<1$. Then

$$
P_{s}^{(p)} \cong e^{-i k \rho_{0} S_{p^{2}} / 2}\left\{\int_{0}^{\psi} \cos \mu \phi \cos \nu \phi d \phi+\frac{i k S_{p} \rho_{0}}{2} \int_{0}^{\psi} \phi^{2} \cos \mu \phi \cos \nu \phi d \phi\right\}
$$

where $\mu=k \rho_{0} C_{p} \cong k h C_{p} / \psi$ and $\nu=\nu_{s}$. In view of

$$
\nu \tan \nu \psi=i \epsilon \omega \rho_{0} Z_{0}
$$

and

it follows that (when $Z_{0}=Z_{1}$ )

$$
\mu \tan \mu \psi=i \epsilon \omega \rho_{0} Z_{1}
$$

$$
\int_{0}^{\psi} \cos \mu \phi \cos \nu \phi d \phi=\left\{\begin{array}{l}
\frac{\psi}{2}\left[1+\frac{\sin 2 \mu \psi}{2 \mu \psi}\right] \text { for } \nu=\mu \\
0 \text { for } \nu \neq \mu .
\end{array}\right.
$$

The second integral of eq (24) can be reduced in the following way 


$$
\begin{aligned}
\int_{0}^{\psi} \phi^{2} \cos \mu \phi \cos \nu \phi d \phi & =\frac{1}{2} \int_{0}^{\psi} \phi^{2} \cos (\mu-\nu) \phi d \phi+\frac{1}{2} \int_{0}^{\psi} \phi^{2} \cos (\mu+\nu) \phi d \phi \\
= & \frac{1}{2(\mu-\nu)^{3}} \int_{0}^{(\mu-\nu) \psi} x^{2} \cos x d x+\frac{1}{2(\mu+\nu)^{3}} \int_{0}^{(\mu+\nu) \psi} x^{2} \cos x d x \\
= & \frac{1}{2(\mu-\nu)^{3}}\left[2(\mu-\nu) \psi \cos (\mu-\nu) \psi+\left[(\mu-\nu)^{2} \psi^{2}-2\right] \sin (\mu-\nu) \psi\right] \\
& \quad+\frac{1}{2(\mu+\nu)^{3}}\left[2(\mu+\nu) \psi \cos \left(\mu+\nu, \psi+\left[(\mu+\nu)^{2} \psi^{2}-2\right] \sin (\mu+\nu) \psi\right] .\right.
\end{aligned}
$$

This latter result is usable for $\mu \neq \nu$. When $\mu=\nu$ the integral may be handled in the manner

$$
\begin{aligned}
\int_{0}^{\psi} \phi^{2} \cos \nu \phi d \phi=\frac{1}{2} \int_{0}^{\psi} \phi^{2}(1+\cos 2 \mu \phi) d \phi= & \frac{\psi^{3}}{6}+\frac{1}{(2 \mu)^{3}} \int_{0}^{2 \mu \phi} x^{2} \cos 2 x d x \\
& =\frac{\psi^{3}}{6}+\frac{1}{8 \mu^{3}}\left[(2 \mu \psi) \cos (2 \mu \psi)+\left[2(\mu \psi)^{2}-1\right] \sin (2 \mu \psi)\right] .
\end{aligned}
$$

While this is essentially the solution of the problem it is desirable to make some further simplifications to permit discussion. Since the important modes at VLF are near grazing we can approximate $\mu$ and $\nu$ as follows [Wait, 1960]

$$
\begin{aligned}
& \mu \simeq \frac{\pi}{\psi}\left(p-\frac{1}{2}\right), \quad p=1,2,3 \ldots \\
& \nu=\nu_{s}=\frac{\pi}{\psi}\left(s-\frac{1}{2}\right), \quad s=1,2,3 \ldots
\end{aligned}
$$

Thus

$$
\begin{aligned}
& \int_{0}^{\psi} \cos \mu \phi \cos \nu \phi d \phi=\left\{\begin{array}{l}
\frac{\psi}{2} \text { for } p=s \\
0 \text { for } p \neq s .
\end{array}\right. \\
& \begin{aligned}
\int_{0}^{\psi} \phi^{2} \cos \mu \phi \cos \nu \phi d \phi & =\frac{\psi}{(\mu-\nu)^{2}} \cos \pi(p-s)+\frac{\psi}{(\mu+\nu)^{2}} \cos \pi(p+s) \\
& =\frac{\psi^{3}}{\pi^{2}}\left[\frac{(-1)^{p-s}}{(p-s)^{2}}+\frac{(-1)^{p+s-1}}{(p+s-1)^{2}}\right] \text { for } p \neq s .
\end{aligned}
\end{aligned}
$$

On the other hand, if $p=s$, the integral is given by

$$
\int_{0}^{\psi} \phi^{2} \cos \mu \phi l \phi=\frac{\psi^{3}}{6}-\frac{\psi^{3}}{\pi^{2}(2 p-1)^{2}} .
$$

We are now in a position to write down some relatively simple formulae which describe the transition. First, we see that

$$
P_{p}^{(p)} \cong \frac{\psi}{2}\left[1+O\left(\psi^{2}\right)\right]
$$

where $O\left(\psi^{2}\right)$ is a quantity which is of second-order smallness in $\psi$. Consequently

$$
A_{p}^{(p)}=\frac{1}{H_{\nu_{s}}^{(2)}\left(k \rho_{0}\right)}\left[1+O\left(\psi^{2}\right)\right] .
$$

In the case of the modes where $p \neq s$,

and therefore

$$
P_{s}^{(p)}=\frac{i k \rho_{0}}{2} \frac{\psi^{3}}{\pi^{2}}\left[\frac{(-1)^{p-s}}{(p-s)^{2}}+\frac{(-1)^{p+s-1}}{(p+s-1)^{2}}\right]
$$

$$
A_{s}^{(p)}=\frac{i k \rho_{0} \psi^{2}(-1)^{s-p}}{\pi^{2} H_{\nu_{s}}^{(2)}\left(k \rho_{0}\right)}\left[\frac{1}{(p-s)^{2}}-\frac{1}{(p+s-1)^{2}}\right] \text {. }
$$


A measure of the mode conversion is expressed by the ratio

$$
\frac{P_{s}^{(p)}}{P_{p}^{(p)}} \cong \frac{i k h \psi}{\pi^{2}}\left[\frac{1}{(p-s)^{2}}-\frac{1}{(p+s-1)^{2}}\right](-1)^{s-p} .
$$

When the incident mode is of order $p=1$, this ratio is simply expressed by

and for $s=2$

$$
\frac{P_{s}^{(1)}}{P_{1}^{(1)}} \cong \frac{i k h \psi}{\pi^{2}}\left[\frac{1-2 s}{s^{2}(s-1)^{2}}\right](-1)^{s}
$$

$$
\frac{P_{2}^{(1)}}{P_{1}^{(1)}} \cong-\frac{i k h \psi}{\pi^{2}} \cdot \frac{3}{4}
$$

We are now in a position to calculate the field in the parallel plate region for $x>b$. Here the field may also be written as a sum of modes in the manner

$$
H_{z}=\sum_{n} B_{n} e^{-i k(x-b) S_{n}} \cos \left(k C_{n} z\right)
$$

for $0<z<h+\Delta h$. The modal equation in this case is

$$
C_{n} \tan \left[k C_{n}(h+\Delta h)\right]=i Z_{2} / \eta_{0}
$$

and in view of the orthogonality of the modes

$$
B_{n}=\frac{\int_{0}^{h+\Delta h}\left[H_{z}(x=b)\right] \cos \left(k C_{n} z\right) d z}{\frac{\hat{h}}{2}\left[1+\frac{\sin 2 k C_{n} \hat{h}}{2 k C_{n} \hat{h}}\right]},
$$

where $\hat{\mathrm{h}}=h+\Delta h$. Following earlier reasoning, the field $H_{z}$ over the aperture $x=b$ is now approximated by the field incident from the wedge transition region. Thus

where

$$
H_{z}(x=b) \cong H_{z}\left(\rho_{0}^{\prime}, \phi\right) e^{i x_{0}^{\prime} k S_{n}}
$$

and

$$
\begin{aligned}
\rho_{0}^{\prime} & =\left[b^{2}+(h+\Delta h)^{2}\right]^{1 / 2} \\
x_{0}^{\prime} & =\left(\rho_{0}^{\prime}-z^{2}\right)^{\frac{1}{2}}-\rho_{0}^{\prime} \cos \psi \\
& \simeq \rho_{0}^{\prime}\left(\frac{\psi^{2}}{2}-\frac{\phi^{2}}{2}\right)
\end{aligned}
$$

$$
H_{z}\left(\rho_{0}^{\prime}, \phi\right)=\sum_{s} A_{s}^{(p)} H_{\nu_{s}}^{(2)}\left(k \rho_{0}^{\prime}\right) \cos \nu_{s} \phi
$$

Again, for the sake of simplicity, one may choose $Z(b)=Z_{2}$ so there is no discontinuity in the surface impedance.

\section{Summary of Final Results}

We are now in a position to summarize and restate the results obtained above. The fields in the three waveguide regions have the following (approximate) forms

$$
\begin{aligned}
& H_{z}=e^{-i k S_{p}^{\prime} x} \cos \left(k C_{p}^{\prime} z\right) \quad \text { for } x<0, \\
& H_{z} \cong\left(\frac{h}{h+x \psi}\right)^{\frac{1}{2}} \sum_{s} \alpha_{s} e^{-i k \int_{0}^{x} S_{s}(x) d x} \cos \left[k C_{s}(x) z\right] \quad \text { for } 0<x<b, \\
& H_{z} \cong\left(\frac{h}{h+b \psi}\right)^{\frac{1}{2}} \sum_{q} \beta_{q} e^{-i k S_{q}^{\prime \prime}(x-b)} \cos \left(k C_{q}^{\prime \prime} z\right) \quad \text { for } x>b .
\end{aligned}
$$


The respective modal equations are

$$
\begin{aligned}
& C^{\prime} \tan \left(k C^{\prime} h\right)=i Z_{1} / \eta_{0}, \\
& C(x) \tan [k C(x) h(x)]=i Z(x) / \eta_{0}, \\
& C^{\prime \prime} \tan k C^{\prime \prime}(h+\Delta h)=i Z_{2} / \eta_{0} .
\end{aligned}
$$

The coefficients $\alpha_{s}$ and $\beta_{q}$ are expressible in terms of Fresnel integrals which are functions of the angle $\psi, b, Z_{1}, Z_{2}$, and $Z(x)$ as discussed in previous sections. Furthermore, in order to assure orthogonality of the modes in the wedge region, it was necessary to choose

and

$$
\left.\begin{array}{l}
Z(x)=Z_{0} \frac{a}{a+x} \\
h(x)=h+\frac{x}{b} \Delta h
\end{array}\right\} \text { for } 0<x<b .
$$

It can be expected that the nature of the coefficients $\alpha_{s}$ and $\beta_{q}$ are not significantly influenced by the finite values of the surface impedances $Z_{1}, Z(x)$, and $Z_{2}$. This is particularly so in the case of grazing modes where the phase shift on reflection at the upper boundary is near $\pi$. Therefore

and

$$
\alpha_{p} \cong 1
$$

\[ \begin{aligned} & \alpha_{s} \cong i k h \psi\left[\frac{1}{(p-s)^{2}}\right.\left.-\frac{1}{(p+s+1)^{2}}\right](-1)^{s-p}, \\ & s \neq p . \\ & \text { If } \quad p=1, \\ & \alpha_{1} \cong 1\end{aligned} \]

and

for

$$
\alpha_{s} \cong \frac{i k h \psi}{\pi^{2}}\left[\frac{1-2 s}{s^{2}(s-1)^{2}}\right](-1)^{s}
$$

$$
s=2,3,4 \ldots
$$

Now at the junction at $x=b$, the situation is very similar. Thus $\beta_{p} \cong 1$ and $\beta_{s} \cong-\alpha_{s}$ for $s \neq p$. This implies, of course, that reconversion from modes of order $s(\neq p)$ back to the mode of order $p$ are neglected. In view of our previous assumption about the smallness of $\psi$, this is entirely justified.

We see from the present analysis that the mode conversion is probably an extremely small effect and can be neglected in most cases of practical interest. Indeed, experimental data on amplitude and phase variations do not appear to have any pronounced and reproducible features which could be attributed to mode conversion at a day/night transition. ${ }^{2}$ However, local ionospheric disturbances may well produce abrupt height changes which could lead to effective values of $\psi$ which are not small. Further effort is being directed toward a better understanding of these phenomena.

I thank Mrs. Alyce M. Conda for pointing out a number of copying errors. I am also indebted to Prof. J. E. Lindsay for a number of valuable comments.

${ }^{2}$ A. G. Jean (personal communication) indicates that there is a consistent phase distortion for the path NBA (Panama) to Boulder. It is possible this could be attributed to the interaction with the spurious second mode produced at the transition boundary. 
Evaluation of

\section{Appendix}

$$
I(\mu, \nu, \alpha, \psi)=\int_{0}^{\psi} \cos \mu \phi \cos \nu \phi e^{i \alpha \phi^{2}} d \phi
$$

The above integral can be transformed in the following manner

$$
\begin{aligned}
& \frac{1}{2} \int_{0}^{\psi} \cos (\mu-\nu) \phi e^{i \alpha \phi^{2}} d \phi+\frac{1}{2} \int_{0}^{\psi} \cos (\mu+\nu) e^{i \alpha \phi^{2} d \phi} \\
& =\frac{1}{4} \int_{0}^{\psi} e^{i(\mu-\nu) \phi} e^{i \alpha \phi^{2}} d \phi+\frac{1}{4} \int_{0}^{\psi} e^{-i(\mu-\nu) \phi} e^{i \alpha \phi^{2}} d \phi+\ldots \\
& =\frac{1}{4} e^{-i \frac{(\mu-\nu)^{2}}{4 \alpha}} \int_{0}^{\psi} e^{i \alpha\left(\phi^{2}+\frac{\mu-\nu}{\alpha} \phi+\frac{(\mu-\nu)^{2}}{4 \alpha^{2}}\right)} d \phi+\ldots \\
& =\frac{1}{4} e^{-i \frac{(\mu-\nu)^{2}}{4 \alpha}} \int_{0}^{\psi} e^{i \alpha\left(\phi+\frac{\mu-\nu}{2 \alpha}\right)^{2}} d \phi+\ldots \\
& =\frac{1}{4} e^{-i \frac{(\mu-\nu)^{2}}{4 \alpha}} \int_{x_{1}}^{x_{2}} e^{i \frac{\pi}{2} x^{2}}\left(\frac{\pi}{2 a}\right)^{\frac{1}{2}} d x+\ldots
\end{aligned}
$$

where

$$
x_{1}=\left(\frac{2 \alpha}{\pi}\right)^{\frac{1}{2}}\left(\frac{\mu-\nu}{2 \alpha}\right)
$$

and

$$
x_{2}=\left(\frac{2 \alpha}{\pi}\right)^{\frac{1}{2}}\left(\psi+\frac{\mu-\nu}{2 \alpha}\right) .
$$

Thus

$$
\begin{aligned}
I(\mu, \nu, \alpha, \psi)=\frac{e^{-i \frac{(\mu-\nu)^{2}}{4 \alpha}}}{4}\{F & {\left.\left[\left(\frac{2 \alpha}{\pi}\right)^{\frac{1}{2}}\left(\psi+\frac{\mu-\nu}{2 \alpha}\right)\right]+F\left[\left(\frac{2 \alpha}{\pi}\right)^{\frac{1}{2}}\left(\psi-\frac{\mu-\nu}{2 \alpha}\right)\right]\right\}\left(\frac{\pi}{2 \alpha}\right)^{\frac{1}{2}} } \\
+ & \frac{e^{-i \frac{(\mu+\nu)^{2}}{4 \alpha}}}{4}\left\{F\left[\left(\frac{2 \alpha}{\pi}\right)^{\frac{1}{2}}\left(\psi+\frac{\mu+\nu}{2 \alpha}\right)\right]+F\left[\left(\frac{2 \alpha}{\pi}\right)^{\frac{1}{2}}\left(\psi-\frac{\mu+\nu}{2 \alpha}\right)\right]\right\}\left(\frac{\pi}{2 \alpha}\right)^{\frac{1}{2}}
\end{aligned}
$$

where $F(x)=\int_{0}^{x} e^{i \frac{\pi}{2} x^{2}} d x$ is a Fresnel integral.

\section{References}

Furutsu, K., Propagation of electromagnetic waves over a flat earth across a boundary, Journal of Radio Research Laboratories (Tokyo) 2, pp. 1-30 (Jan. 1955).

Lauter, E. A., and K. Sprenger, Nächtliche Ionisationstörungen der tiefen Ionosphäre, Z. Meteorol. 6, p. $151(1952)$.

Solymar, L., Overmoded waveguides, Electronic and Radio Engineer, pp. 426-428 (Nov. 1959).

Wait, J. R., Electromagnetic radiation from cylindrical structures (Pergamon Press, London and New York, 1959). (Many other related references are given here.)

Wait, J. R., Terrestrial propagation of very-low-frequency radio waves, J. Research NBS 64D (Radio Prop.) No. 2, 153-204 (Mar.-Apr. 1960).

(Paper 66D4-207) 